\title{
Aplicação do Método de Houbolt na formulação de Elementos de Contorno com Dupla Reciprocidade
}

\author{
Carlos Andrés Reyna Vera-Tudela
}

Universidade Federal Rural do Rio de Janeiro, Instituto de Ciências Exatas, Departamento de Matemática, Programa de Mestrado em Modelagem Matemática e Computacional, Rodovia BR 465, Km 07, Zona Rural, Seropédica 23897-000, Rio de Janeiro, Brazil. E-mail: carlos.reyna.veratudela@gmail.com

Vera-Tudela C.A.R. (2020) Aplicação do método de Houbolt na formulação de elementos de contorno com dupla reciprocidade. Pesquisa e Ensino em Ciências Exatas e da Natureza, 4: e1558.

http://dx.doi.org/10.29215/pecen.v4i0.1558

Editor acadêmico: Saulo Pomponet Oliveira. Recebido: 25 maio 2020. Aceito: 13 novembro 2020. Publicado: 25 novembro 2020

Resumo: Neste trabalho, a formulação da dupla reciprocidade no método dos elementos de contorno é revisitada numa aplicação a problemas de campo vetorial como são os problemas elastodinâmicos. São estudados problemas onde a resposta dinâmica é resolvida utilizando o esquema de Houbolt para a integração temporal. Dois exemplos são apresentados, resolvidos e o desempenho deles é comparado com soluções analíticas. Finalmente é analisado o efeito da introdução de pontos internos no domínio do corpo.

Palavras chave: Esquema de Houbolt, método dos elementos de contorno, dupla reciprocidade.

\section{Application of the Houbolt method in the boundary element formulation with dual reciprocity}

Abstract: This work revisits the double reciprocity formulation in the boundary element method through its application to vector field problems such as elastodynamic problems. Problems are studied where the dynamic response is solved using the Houbolt scheme for temporal integration. Two examples are presented, solved and their performance is compared with analytical solutions. Finally, the effect of introducing internal points into the body domain is analyzed.

Key words: Houbolt scheme, boundary element method, dual reciprocity.

\section{Introdução}

$\mathrm{Na}$ engenharia e nas ciências em geral, existe um processo contínuo de procura por novas técnicas, novos métodos de solução de problemas, e formas diferentes de encarar problemas teóricos ou práticos. Muitas vezes esta procura está direcionada na evolução, no aperfeiçoamento, na melhora contínua, que é fundamento do crescimento da sociedade.

A história da humanidade tem muitos exemplos que mostram esta evolução; e um caso muito próximo está relacionado com o computador. Todos lembram aquelas máquinas enormes, com longos tempos de processamento e, segundo a visão atual, recursos muito limitados. $\mathrm{O}$ objetivo inicial, com este exemplo comum à maioria das pessoas, é mostrar como o progresso atual do computador está relacionado com o estudo contínuo, as melhoras e o desenvolvimento de técnicas paralelas, de tal forma que a união de tudo isso forma o conjunto de máquinas, programas, etc., com recursos inimagináveis até poucos anos atrás. E hoje têm-se consciência que, para o futuro próximo, não há limites na criatividade humana e muito ainda está por vir.

Junto com o desenvolvimento do computador, a engenharia acompanha este progresso, com intensidade tão singular como nunca experimentou em todas as épocas anteriores. Assim, junto ao aprimoramento dos equipamentos, houve a necessidade de desenvolvimento de ponderosas ferramentas matemáticas e computacionais, que explorassem convenientemente a potencialidade das novas máquinas. 
Dentre as muitas técnicas que poderiam ser mencionadas, destaca-se em primeiro lugar, o Método dos Elementos Finitos (MEF) (Bathe 1996), que tem especial importância em razão da sua enorme difusão e receptividade; e segundo, o Método das Diferenças Finitas (MDF) (Burden \& Faires 2008), com grande aceitação e desenvolvimento.

Posteriormente, surgiu uma técnica, chamada de Método dos Elementos de Contorno (MEC) (Brebbia et al. 2012), ganhando notoriedade graças a uma série de características vantajosas, como a redução em uma dimensão na representação do problema (Li et al. 2018; Ye et al. 2018; Wang et al. 2019), adequação a problemas com regiões infinitas (Morovati \& Malek 2015; Klaseboer et al. 2019; Parvanova et al. 2020), melhor captação de concentração de tensões (Li et al. 2015; Lei et al. 2017; Mukhtar 2017), entre outras.

Basicamente, pode-se dizer que o Método dos Elementos de Contorno (Brebbia \& Dominguez 1994; Trevelyan 1994; Katsikadelis 2002) transforma equações diferenciais parciais, que governam o domínio do problema, em equações integrais envolvendo somente valores de contorno, após a introdução de funcões de ponderação denominadas soluções fundamentais. Desta forma, a dimensão do problema é reduzida de uma unidade e somente o contorno do domínio precisa ser discretizado. Esta é uma das grandes vantagens do método, porque ao discretizar somente o contorno vai-se ter uma menor entrada de dados, operações numéricas mais simples e, dependendo da extensão do problema, um menor dispêndio computacional.

O Método da Dupla Reciprocidade é um procedimento apresentado por D. Nardini e C. A. Brebbia em 1982 (Nardini \& Brebbia 1983) para resolver problemas transientes usando soluções fundamentais estáticas, mas que se revelou bastante adequado e eficaz na solução de problemas com ações no domínio. $\mathrm{O}$ objetivo da técnica é transformar a integral de domínio do termo "forçante", em uma integral de contorno (Partridge et al. 1991; Cheng et al. 2001). A estratégia é de fazer a substituição da grandeza que tem as características de ação de domínio pelo produto de duas outras variáveis, ficando apenas uma delas dependente das grandezas espaciais. A Dupla Reciprocidade surge, então, como uma formulação alternativa do MEC, que mediante o uso de funções auxiliares permite a transformação das integrais de domínio em integrais de contorno, de acordo com os procedimentos clássicos do método.

$\mathrm{O}$ presente trabalho ilustra o método de dupla reciprocidade em problemas lineares de elastodinâmica, revisando sua formulação e implementação computacional, assim como apresentando a resolução de dois exemplos bidimensionais. Para a aproximação temporal, optouse pelo esquema de Houboult (Houbolt 1950; Guendelman 2008).

\section{Metodologia}

\section{O Método da Dupla Reciprocidade}

A Equação de Navier, que é uma equação de movimento em termo de deslocamentos, é representada como se vê na equação seguinte:

$$
G u_{j}, i i+\frac{G}{1-2 v} u_{i}, i j=\rho \ddot{u}_{j}
$$

onde $\ddot{u}_{j}$ representa o efeito da inercia no corpo, $u$ é o deslocamento, $G$ é o módulo de cisalhamento e $v$ é o coeficiente de Poisson. Na equação (1) e em todas as equações que se seguem no texto é utilizada a notação de Einstein ou notação indicial (Malvern 1969).

Na equação de Navier são aplicados todos os procedimentos clássicos do MEC (Nardini \& Brebbia 1983), obtendo-se:

$$
\begin{aligned}
& C_{i j}(\xi) u_{j}(\xi)+\int_{\Gamma} p_{i j}^{*}(\xi, x) u_{j}(x) d \Gamma(x)- \\
& \qquad \int_{\Gamma} u_{i j}^{*}(\xi, x) p_{j}(x) d \Gamma(x)=\int_{\Omega} u_{i j}^{*}(\xi, x) \rho \ddot{u}_{j}(x) d \Omega
\end{aligned}
$$


onde $\Gamma$ representa o contorno do problema; $u_{i j}^{*}$ na formulação tradicional corresponde à solução fundamental de Kelvin; $p_{i j}^{*}$ corresponde à força de superfície fundamental; $C_{i j}$ é um coeficiente relacionado à geometria do corpo e $\xi$ é o ponto fonte.

A solução fundamental para o problema de estado plano num meio elástico infinito é dada pela expressão seguinte (Brebbia et al. 2012),

$$
u_{i j}^{*}(\xi, x)=\frac{-1}{8 \pi(1-v)}\left[(3-4 v) \ln (r) \delta_{i j}-r,{ }_{i} r, j\right]
$$

e

$$
p_{i j}^{*}(\xi, x)=\frac{-1}{4 \pi(1-v) r}\left\{\left[(1-2 v) \delta_{i j}+2 r,{ }_{i} r, j\right] \frac{\partial r}{\partial n}-(1-2 v)\left(r,{ }_{i} n_{j}-r,{ }_{j} n_{i}\right)\right\}
$$

onde $r$ é a distância do ponto de aplicação da carga ao ponto em consideração. A Solução Fundamental é um tipo de função utilizada para resolver equações diferenciais não-homogêneas sujeitas a condições iniciais ou condições de contorno determinadas, e descreve a resposta do sistema físico ao delta de Dirac, que representa uma carga concentrada aplicada no ponto $\mathrm{x}$ (magnitude unitária).

O Método da Dupla Reciprocidade (Nardini \& Brebbia 1983) é introduzido de forma a expressar o efeito de domínio como uma integral de contorno. Para isso, representa-se este termo por um somatório de funções na forma indicial seguinte:

$$
\ddot{u}_{j}(x)=\ddot{\alpha}_{j}^{m} f^{m}=\ddot{\alpha}_{j}^{1} f^{1}+\ddot{\alpha}_{j}^{2} f^{2}+\ldots+\ddot{\alpha}_{j}^{n} f^{n}
$$

onde os pontos $x$ são pontos de colocação, $f$ é uma função de aproximação de base radial e $\left\{\ddot{\alpha}_{j}^{1}, \ddot{\alpha}_{j}^{2}, \ldots, \ddot{\alpha}_{j}^{n}\right\}$ é um conjunto de coeficientes iniciais desconhecidos.

Desta forma, a integral de domínio passa a ser escrita como:

$$
\int_{\Omega} u_{i j}^{*}(\xi, x) \rho \ddot{u}_{j}(x) d \Omega=\rho \ddot{\alpha}_{j}^{m} \int_{\Omega} f^{m}(x) u_{i j}^{*}(\xi, x) d \Omega .
$$

Para transformar o lado direito da equação (6) numa integral de contorno, é necessário reescrever $f^{m}(x)$ em termos de uma função auxiliar $\psi_{n j}^{m}$ que precisa satisfazer à equação de Navier com o termo de domínio sendo concentrado no contorno como segue:

$$
G \psi_{n j, i i}^{k}+\frac{G}{1-2 v} \psi_{n i, i j}^{k}=\delta_{n j} f^{k}
$$

Substituindo a equação (7) na equação (6), e verificando que, por analogia as mesmas operações já desenvolvidas na formulação tradicional podem ser empregadas, obtem-se:

$$
C_{i j}^{l} u_{j}^{l}+\int_{\Gamma} p_{i j}^{*} u_{j} d \Gamma-\int_{\Gamma} u_{i j}^{*} p_{j} d \Gamma=\left(C_{i j}^{l} \psi_{n j}^{l m}+\int_{\Gamma} p_{i j}^{*} \psi_{n j}^{m} d \Gamma-\int_{\Gamma} u_{i j}^{*} \eta_{n j}^{m} d \Gamma\right) \rho \ddot{\alpha}_{m}^{n}
$$

Nesta expressão o termo $\eta_{n j}^{m}$ corresponde às forças de superfície recíprocas, referents à função auxiliar $\psi_{n j}^{m}$, correspondente aos deslocamentos.

A função auxiliar de interpolação é dada pela seguinte expressão (Partridge et al. 1991):

$$
\psi_{n j}=\frac{1}{30(1-v) \mu}\left[\left(3-\frac{10 v}{3}\right) \delta_{n j}-r, n,,_{j}\right] r^{3}
$$




$$
\eta_{n j}=\frac{1}{15(1-v)}\left[(4-5 v) r_{n} v_{j}-(1-5 v) r,{ }_{j} v_{n}+\left[(4-5 v) \delta_{n j}-r,{ }_{j} r_{n}\right] \frac{\partial r}{\partial v}\right] r^{2}
$$

onde $v$ representa o vetor normal externo ao corpo e $\frac{\partial r}{\partial v}=v_{j} r_{, j}$.

\section{Implementação numérica}

Uma vez obtida a equação integral de contorno é preciso discretizá-la para então resolvêla (Vera-Tudela 2003). Para a discretização da equação (8), divide-se o contorno em $j$ elementos $\Gamma_{j}$. A esses elementos estão associados valores nodais de deslocamentos e forças de superfície, em função dos quais a variação destas grandezas dentro do elemento depende das funções de interpolação $N$ utilizadas e que por sua vez dependem do número de nós funcionais $n$ dos elementos. Desta forma, pode-se expressar os valores de deslocamentos $u_{j}$ da equação (8) na forma matricial seguinte:

$$
u_{j}=\left\{\begin{array}{l}
u_{1} \\
u_{2}
\end{array}\right\}=N u^{(n)}
$$

e para forças de superfície $p_{j}$ :

$$
p_{j}=\left\{\begin{array}{l}
p_{1} \\
p_{2}
\end{array}\right\}=N p^{(n)}
$$

onde

$$
N=\left[\begin{array}{cccccc}
\phi_{1} & 0 & \phi_{2} & 0 & \phi_{3} & 0 \\
0 & \phi_{1} & 0 & \phi_{2} & 0 & \phi_{3}
\end{array}\right]
$$

Também, para elemento quadrático, tem-se:

$$
u^{(n)}=\left\{\begin{array}{l}
u_{1}^{1} \\
u_{1}^{2} \\
u_{2}^{1} \\
u_{2}^{2} \\
u_{3}^{1} \\
u_{3}^{3}
\end{array}\right\}, p^{(n)}=\left\{\begin{array}{l}
p_{1}^{1} \\
p_{1}^{2} \\
p_{2}^{1} \\
p_{2}^{2} \\
p_{3}^{1} \\
p_{3}^{3}
\end{array}\right\}
$$

Observe-se que os procedimentos aplicados sobre $u$ e $p$, também serão realizados sobre $\psi$ e $\eta$. Substituindo as equações (11) e (12) na equação (8) tem-se, 


$$
\begin{aligned}
C u+\sum_{j=1}^{J}\left(\int_{\Gamma_{j}} p^{*} N d \Gamma\right) u^{(n)}-\sum_{j=1}^{J}\left(\int_{\Gamma_{j}} u^{*} N d \Gamma\right) p^{(n)}= \\
{\left[\sum_{j=1}^{J} C \psi^{(n)}+\sum_{j=1}^{J}\left(\int_{\Gamma_{j}} \eta^{*} N d \Gamma\right) \psi^{(n)}-\sum_{j=1}^{J}\left(\int_{\Gamma_{j}} \psi^{*} N d \Gamma\right) \eta^{(n)}\right] \rho \ddot{\alpha}^{n} . }
\end{aligned}
$$

O elemento de contorno $\Gamma_{j}$ varia de acordo com a função de interpolação adotada. Neste trabalho é considerada a função de interpolação quadrática com três nós geométricos por elemento.

Durante a montagem do sistema indicado pela equação (15), cada uma das integrais será calculada numericamente sempre que o ponto fonte $\xi$ não pertencer ao elemento $\Gamma_{j}$ sobre o qual está sendo efetuada a integração. Este cálculo se dará através da integração numérica de Gauss dada por,

$$
\int_{-1}^{1} f(\eta) d \eta=\sum_{k=1}^{N P I} f\left(\eta_{k}\right) w_{k}
$$

onde $\eta_{k}$ é a coordenada adimensional do k-ésimo ponto de integração, $w_{k}$ é o fator de peso associado ao ponto $k$ e NPI é o número total de pontos de integração utilizado. Desta forma, trabalha-se com as parcelas da equação (15) como segue,

$$
\int_{\Gamma_{j}} p^{*} N d \Gamma=\int_{-1}^{1} p^{*} N|J| d \eta \cong \sum_{k=1}^{N P I}|J|_{k} w_{k} N_{k} p_{k}^{*}
$$

e

$$
\int_{\Gamma_{j}} u^{*} N d \Gamma=\int_{-1}^{1} u^{*} N|J| d \eta \cong \sum_{k=1}^{N P I}|J|_{k} w_{k} N_{k} u_{k}^{*}
$$

A equação integral discretizada é aplicada repetidamente considerando o ponto fonte $\xi$ situado coincidentemente com todos os pontos nodais existentes. Um sistema de $2 n$ equações algébricas é gerado e envolve $2 n$ valores nodais de deslocamento e força de superfície. Ainda é necessário levar este sistema para uma forma matricial e para isso coloca-se da forma a seguir,

$$
\sum_{j=1}^{J}\left(\int_{\Gamma_{j}} p^{*} N d \Gamma\right) u^{(n)}=\sum_{j=1}^{J} h_{j} u^{(n)} .
$$

Similarmente

$$
\sum_{j=1}^{J}\left(\int_{\Gamma_{j}} u^{*} N d \Gamma\right) p^{(n)}=\sum_{j=1}^{J} g_{j} p^{(n)}
$$

O sistema fica reduzido na forma a seguir, 


$$
C u^{(n)}+\sum_{j=1}^{J} h_{j} u^{(n)}-\sum_{j=1}^{J} g_{j} p^{(n)}=\left[C \psi^{(n)}+\sum_{j=1}^{J} h_{j} \psi^{(n)}-\sum_{j=1}^{J} g_{j} \eta^{(n)}\right] \rho \ddot{\alpha} .
$$

Resulta, então, um sistema de equações matriciais na forma

$$
\mathbf{H u}-\mathbf{G p}=\rho[\mathbf{H} \psi-\mathbf{G} \eta] \ddot{\alpha}
$$

e da Equação 5, pode-se escrever,

$$
\ddot{\alpha}=\mathbf{F}^{-1} \ddot{\mathbf{u}}
$$

Substituindo a Equação 23 em 22, obtém-se,

$$
\mathbf{H u}-\mathbf{G p}=\rho[\mathbf{H} \psi-\mathbf{G} \eta] \mathbf{F}^{-\mathbf{1}} \ddot{\mathbf{u}}
$$

E chamando-se,

$$
\mathbf{M}=\rho[\mathbf{G} \eta-\mathbf{H} \psi] \mathbf{F}^{-\mathbf{1}}
$$

Tem-se

$$
\mathbf{M u ̈}+\mathbf{H u}=\mathbf{G p} .
$$

Na Equação 26, H e G são as matrizes características do Método dos Elementos de Contorno da Elastostática, enquanto $\mathbf{M}$ representa a matriz de massa.

\section{O Problema da Resposta Dinâmica}

A maneira mais usual para se obter uma resposta no tempo com a equação matricial (Equação 26), é utilizar algum esquema de integração linear (Rama \& Rao 2001). Esses esquemas requerem que o equilíbrio do sistema seja obedecido apenas em instantes discretos durante o processo, entre os quais são admitidas variações lineares ou constantes para os deslocamentos, velocidades e acelerações.

Define-se duas categorias de integração linear no tempo: a primeira, que engloba os Métodos Explícitos ou condicionalmente estáveis (Burden \& Faires 2008), onde o principal método é o da Diferença Central, que depende do intervalo $\Delta t$ para se obter bons resultados; a segunda categoria corresponde aos Métodos Implícitos, que podem ser incondicionalmente estáveis, onde pode-se mencionar os métodos de Houbolt, Wilson e Newmark (Guendelman 2008).

Dos esquemas de integração no tempo mais comumente empregados, o esquema Houbolt (1950) é o que apresenta maior alongamento dos períodos naturais de vibração e intensidade de amortecimento fictício. Esse último efeito é equivalente ao truncamento da influência dos modos superiores na resposta. estável.

O esquema de integração de Houbolt é um algoritmo implícito e incondicionalmente 
Seguindo os princípios definidos para o Método dos Elementos de Contorno, o sistema em estudo é constituído de um domínio $\boldsymbol{\Omega}(x)$, delimitado por um contorno $\Gamma(x)$ sujeito às seguintes condições de contorno:

$$
\left\{\begin{array}{l}
u_{1}(x, t)=\bar{u}_{1}(x, t) \text { em } \Gamma_{1} \\
p_{2}(x, t)=\bar{p}_{2}(x, t) \text { em } \Gamma_{2}
\end{array}\right.
$$

O caráter dinâmico do problema também requer condições iniciais em $\boldsymbol{\Omega}(x)$ do tipo:

$$
\left\{\begin{array}{l}
u(x, 0)=u_{0}(x) \\
\dot{u}(x, 0)=\dot{u}_{0}(x)
\end{array}\right.
$$

O esquema de Houbolt apresenta uma aproximação para velocidades da forma que se mostra a seguir:

$$
\dot{u}_{n+1}=\frac{1}{6 \Delta t}\left[11 u_{n+1}-18 u_{n}+9 u_{n-1}-2 u_{n-2}\right]
$$

e a seguinte aproximação para a aceleração é considerada,

$$
\ddot{u}_{n+1}=\frac{1}{\Delta t^{2}}\left[2 u_{n+1}-5 u_{n}+4 u_{n-1}-u_{n-2}\right]
$$

onde $\Delta t$ é o intervalo de discretização no tempo. Experiências mostram que $\Delta t$ deve obedecer a seguinte relação (Yee 1966),

$$
\Delta t \leq \frac{\Delta L}{c}
$$

onde

$$
c=\sqrt{\frac{G}{\rho}}
$$

e $\Delta L$ é o menor comprimento do elemento de contorno usado na elaboração da malha.

A equação matricial de equilíbrio (26) é expressa em termos do instante $n+1$, da forma seguinte,

$$
\mathbf{M} \ddot{\mathbf{u}}_{n+1}+\mathbf{H} \mathbf{u}_{n+1}=\mathbf{G P} \mathbf{P}_{n+1}
$$

Finalmente, substituindo-se a equação (30) na equação (33), obtém-se a expressão para o esquema de avanço no tempo,

$$
\left[2 \mathbf{M}+\Delta t^{2} \mathbf{H}\right] \mathbf{u}_{n+1}-\Delta t^{2} \mathbf{G p}_{n+1}=5 \mathbf{M} \mathbf{u}_{n}-4 \mathbf{M} \mathbf{u}_{n-1}+\mathbf{M} \mathbf{u}_{n-2}
$$




\section{Resultados e Discussão}

\section{Exemplo 1}

O primeiro teste da formulação consiste da análise de uma viga sob o efeito de um carregamento súbito, seguindo uma distribuição senoidal vertical (Hetenyi 1971; Meirovitch 1975), segundo pode-se observar na Figura 1. Considerando a condição de bi-apoio da viga, este tipo de distribuição especial para o carregamento excita quase que exclusivamente o primeiro modo de vibração da estrutura. A Figura 1 também mostra as medidas geométricas do problema e as condições de apoio no contorno. $\mathrm{O}$ carregamento tem valor nulo nos extremos e, de acordo com a variação senoidal, o valor máximo encontra-se no ponto médio da viga e tem valor unitário. A malha está definida para uma discretização com 70 elementos de contorno quadráticos (144 nós). Tem-se nó duplo nos quatro cantos da viga, módulo de Young e massa específica do material com valores unitários e coeficiente de Poisson nulo (de forma a melhor representar o deslocamento vertical da viga sem o efeito da deformação longitudinal). $\mathrm{O}$ tempo total para o teste é de 150 segundos e o intervalo do incremento de tempo é de 1 segundo $(\Delta t=$ $1)$.

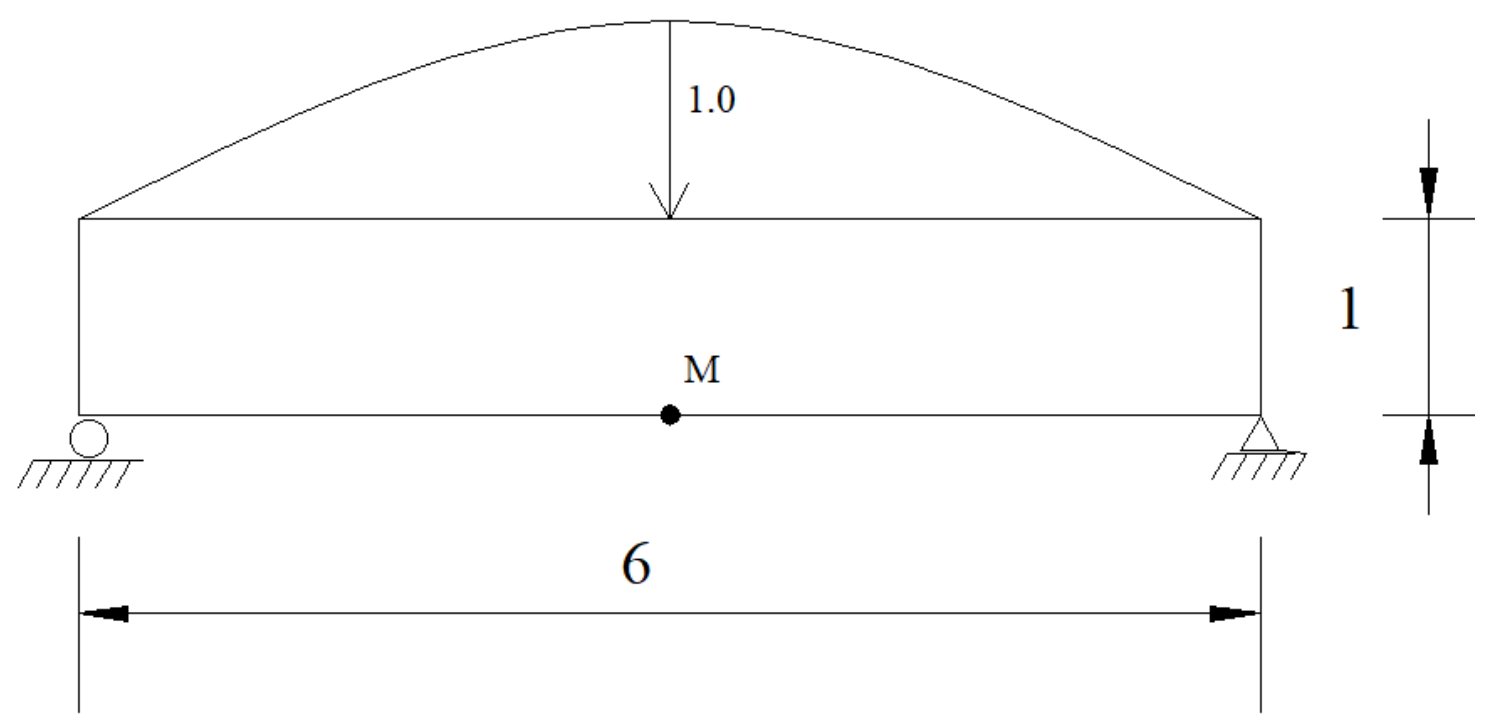

Figura 1. Viga com carregamento de distribuição senoidal.

A análise da formulação para a viga é direcionada no cálculo do deslocamento no ponto M, que é mostrado na Figura 1. Na Figura 2 podem-se observar os deslocamentos no ponto M, onde a resposta tem um comportamento senoidal. O valor máximo do deslocamento é aproximadamente igual a duas vezes o valor do deslocamento estático. A análise dinâmica do problema através da Teoria da Elasticidade, admitindo um estado plano de tensão, não tem solução exata. Mas o exame do mesmo pela teoria unidimensional de vigas mostra que este tipo de carregamento súbito resulta de fato num pico de deslocamento transversal próximo do dobro do valor estático, enquanto a resposta no tempo varia harmonicamente, conforme mostrado. $\mathrm{O}$ período fundamental da estrutura, que pode ser percebido claramente pela curva de resposta numérica, também encontra-se bem próximo do valor analítico dado pela teoria de vibração em vigas (Sayyad \& Ghugal 2016). Este teste foi comparado com o Método das Diferenças Finitas com uma malha de 20 divisões e 148 intervalos de tempo. Desse modo, conclui-se que os resultados obtidos são amplamente satisfatórios. 


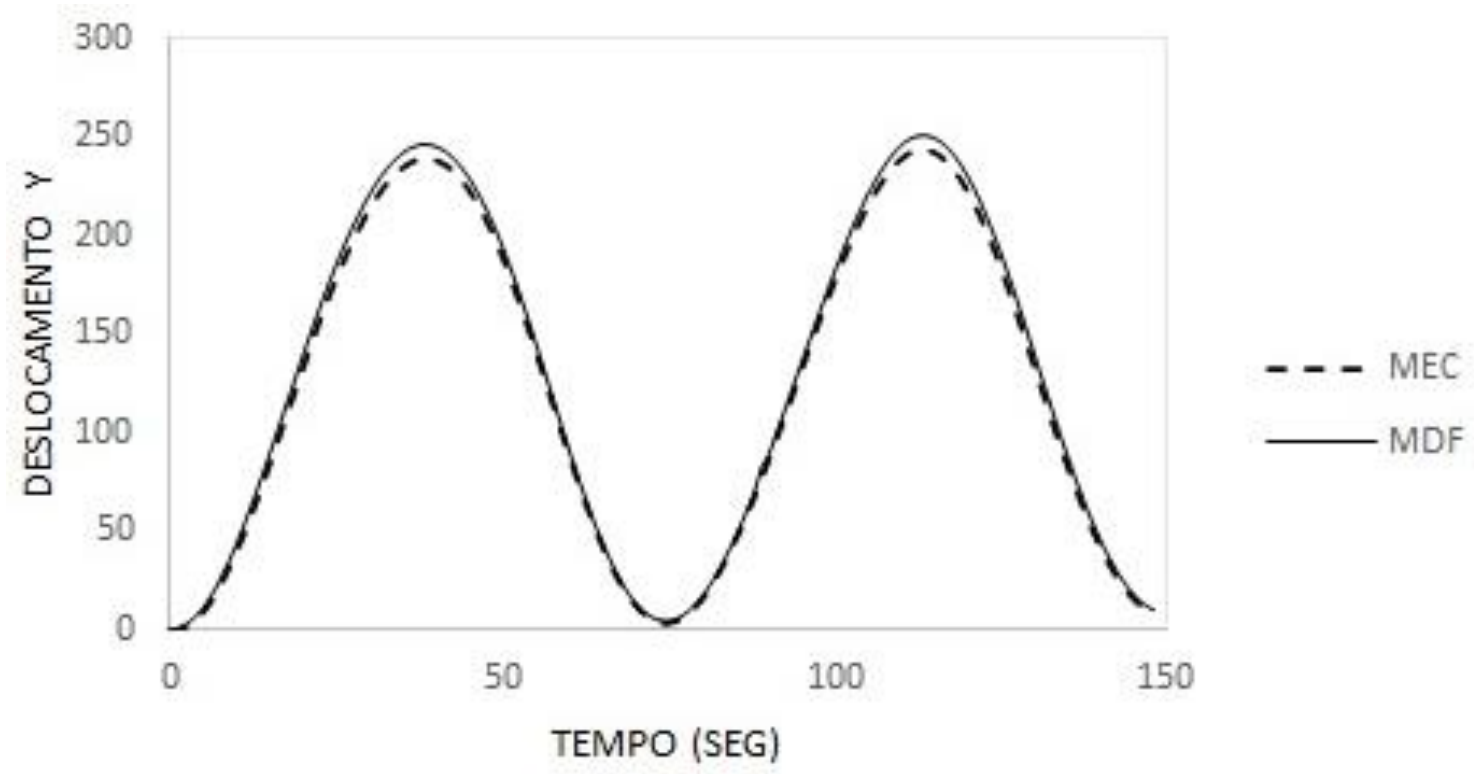

Figura 2. Deslocamento Y no ponto M da viga comparado com um modelo de Diferenças Finitas.

\section{Exemplo 2}

Casos como o mostrado no exemplo anterior, ou então situações onde a viga é submetida a carregamentos uniformes constantes, são muito comuns em análise dinâmica de estruturas, mas excitam basicamente o modo fundamental de vibração. Para uma melhor avaliação da consistência numérica do método é interessante investigar casos onde um grande número de modos de vibração esteja presente. Tais problemas são vulgarmente conhecidos como problemas de propagação de ondas. Assim, neste segundo exemplo são estudados os resultados obtidos para a propagação de ondas longitudinais elásticas em uma barra fixa em uma das suas extremidades e submetida a um carregamento súbito na outra extremidade. Ressalte-se que embora aparentemente simples, a solução numérica deste problema é difícil, pois há participação de todos os modos de vibração (Nogueira \& Loeffler 1991). Há ainda a presença de uma frente de onda bem definida, que se propaga progressivamente ao longo da barra, impondo uma descontinuidade no campo de tensões. A Figura 3 mostra as características geométricas do sistema e o carregamento imposto.

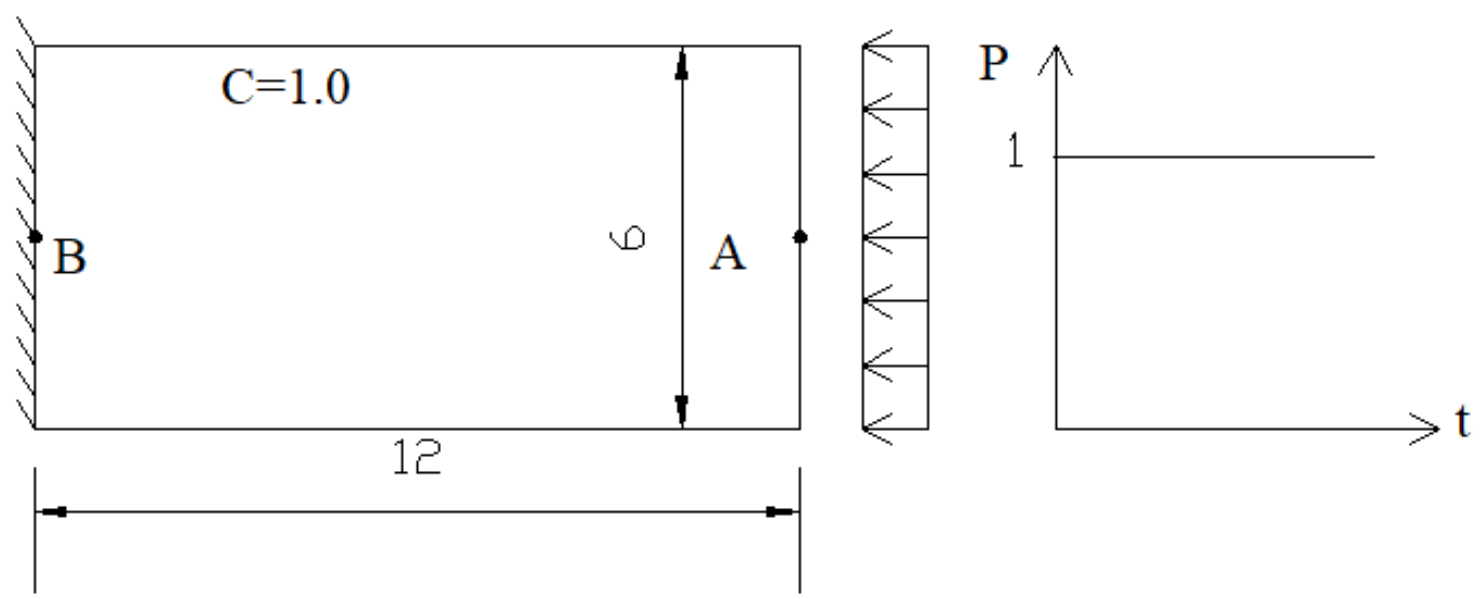

Figura 3. Barra de seção transversal constante axialmente solicitada.

Considera-se uma malha composta de 36 elementos quadráticos (76 nós) com nós duplos nos cantos. As propriedades físicas correspondem a valores unitários do Módulo de Young e da massa específica. A malha tem 30 graus de liberdade, o tempo total do teste é de 160 
segundos e o intervalo do incremento de tempo é $\Delta t=1$ segundo. O coeficiente de Poisson é igual a zero, de modo a melhor simular o comportamento típico da barra sob tração.

É estudado o nó A para deslocamentos e o nó B para tensões. Na Figura 4 mostram-se os resultados de deslocamento para o ponto A, que como pode ser observado, são de boa precisão.

Na Figura 5 são apresentados os resultados deste teste, retratando agora os valores das forças no ponto B.

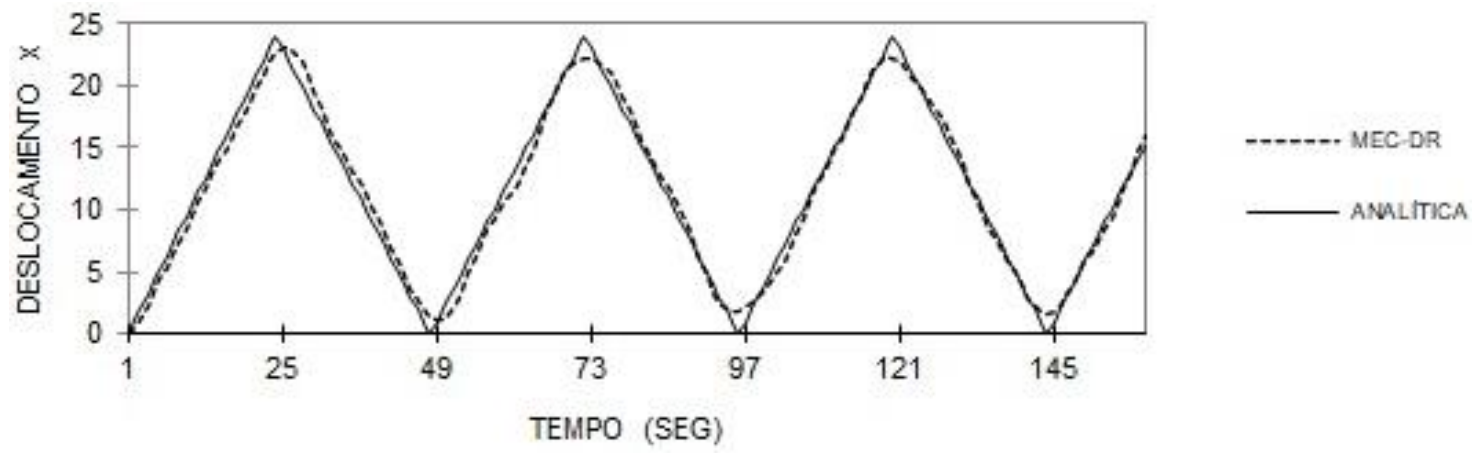

Figura 4. Deslocamento no ponto A para a malha de 36 elementos de contorno.

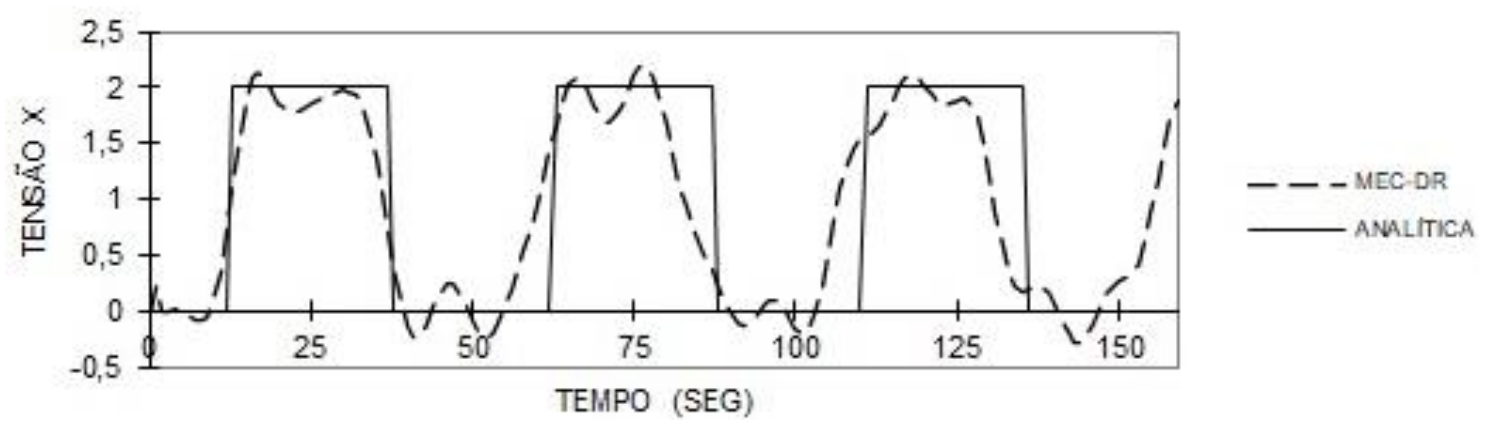

Figura 5. Tensão no ponto B para a malha de 36 elementos de contorno.

Nota-se que as forças são de bem mais difícil representação, pois sua resposta é periodicamente descontínua e também são influenciadas pelos modos de vibração mais elevados, que forçosamente são mal representados na modelagem numérica. Uma vantagem importante está relacionada com o amortecimento fictício próprio do esquema Houbolt, que elimina as oscilações indesejáveis. No entanto, o esquema tende a suavizar os gradientes e encurtar o período de resposta. Outros testes feitos para intervalos de tempo menores e maiores mostram que existe um intervalo onde a representação do fenômeno é satisfatória.

É interessante mostrar agora o efeito da inclusão de pontos internos (Zhao et al. 1994) na barra. Nos casos pertinentes à teoria de campo escalar, a implantação de pontos internos é muito importante. Assim, os próximos testes correspondem a geometrias com 3, 6 e 12 pontos internos, cuja distribuição é mostrada esquematicamente na Figura 6. A Figura 7 mostra os resultados para o teste considerando o deslocamento sobre o ponto A.

Observa-se que a inclusão de pontos internos não mostra uma melhora significativa nos resultados, o que poderia indicar que para a elastodinâmica a sensibilidade do modelo computacional aos pontos internos é bem menor do que na dinâmica escalar.

A Figura 8 apresenta os valores de força para o ponto $\mathrm{B}$ ao longo do tempo. $\mathrm{O}$ mesmo se conclui com relação às forças de superfície, onde a inclusão de pontos internos constitutivos, também chamados pólos, não resultou em melhoria perceptível. Apesar da dificuldade numérica que envolve esse problema (que por essa razão é um caso clássico para testes de técnicas numéricas em dinâmica), percebe-se que melhores resultados poderiam ser obtidos com um esquema de integração passo-a-passo mais eficiente, tal que amortecesse os modos mais altos e espúrios, enquanto produzisse menores distorções nos resultados. 


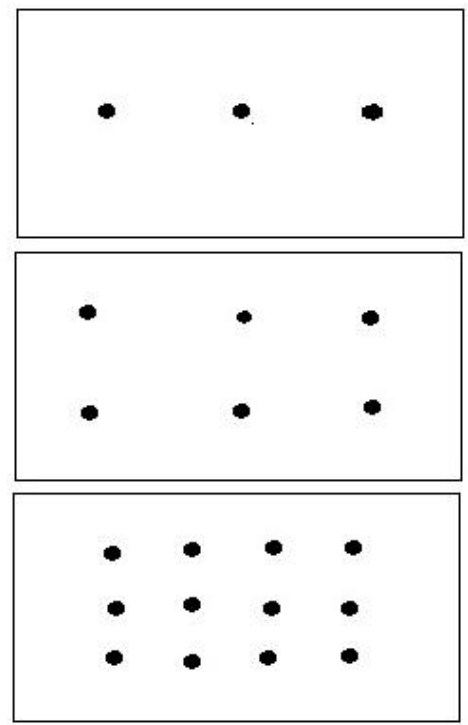

Figura 6. Representação dos pontos internos no interior da malha.
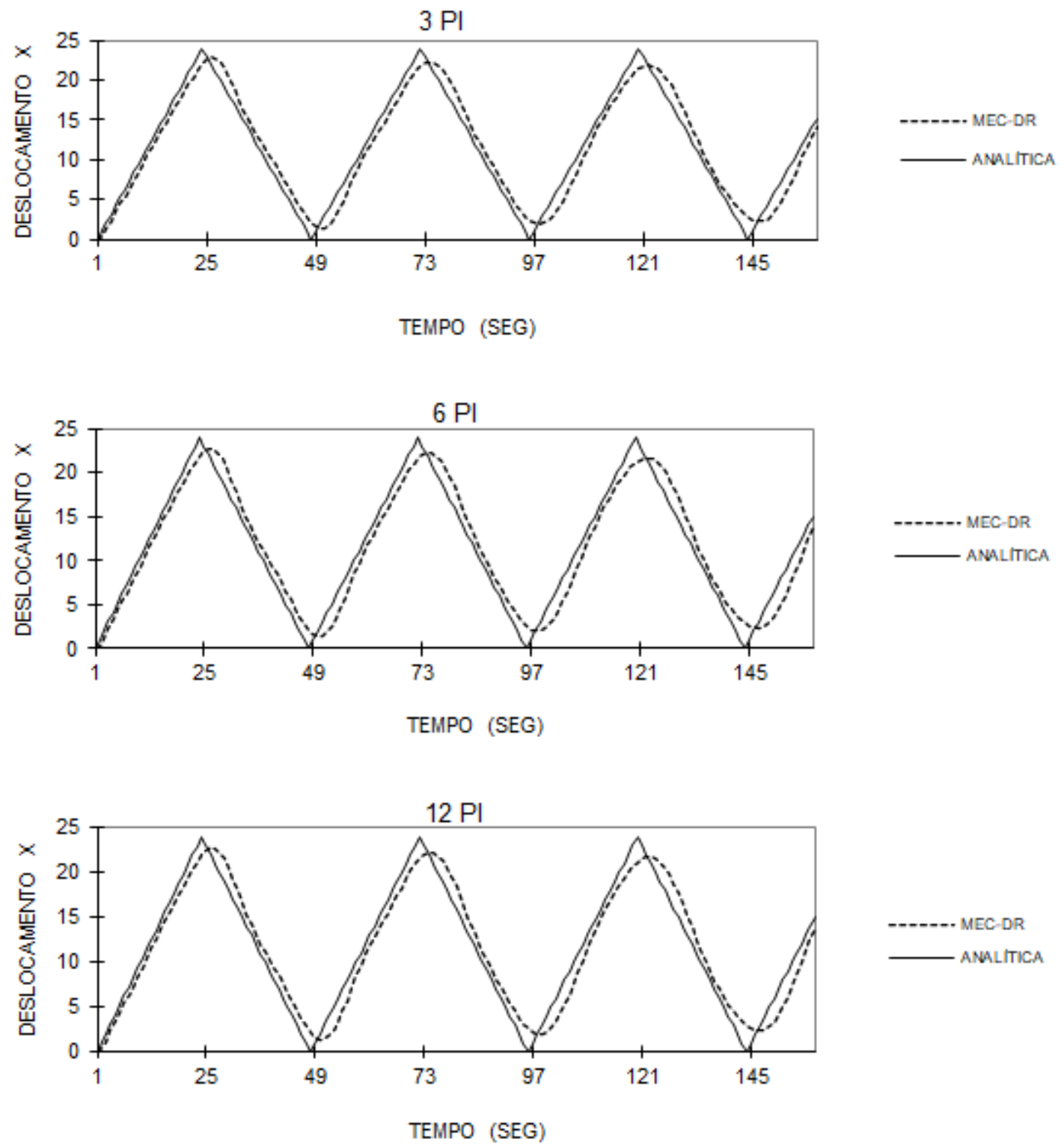

Figura 7. Deslocamento no ponto A, considerando 3, 6 e 12 pontos internos. 

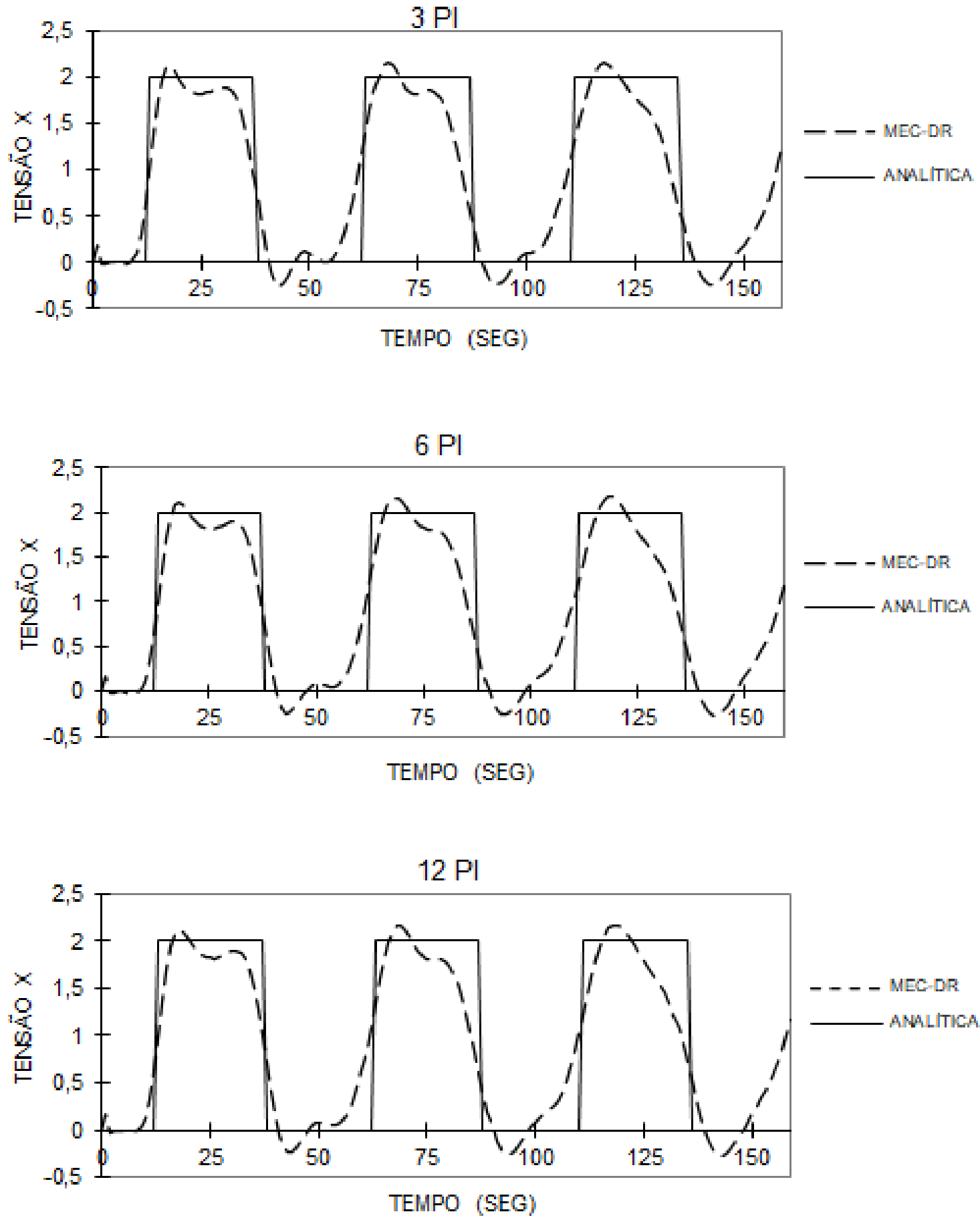

Figura 8. Tensão no ponto B, considerando 3, 6 e 12 pontos internos.

\section{Conclusões}

Pelo exposto, primeiramente verificou-se que a formulação do Método dos Elementos de Contorno com Dupla Reciprocidade mostrou-se consistente, resolvendo satisfatoriamente, ou seja, com precisão plenamente aceitável para engenharia, problemas elastodinâmicos bidimensionais.

No primeiro exemplo onde tem-se um carregamento harmônico ou periódico de baixa frequência é bem mais fácil de simular computacionalmente, pois excita basicamente o modo mais próximo à frequência forçante, cuja representação numérica é bastante boa. $O$ transiente de resposta, de conteúdo modal mais amplo, pode ser desprezado. Por isso, os resultados foram mais precisos. 
No entanto, o Exemplo 2 onde a distribuição do carregamento é localizada e sua aplicação é feita subitamente tem um comportamento bastante difícil de simular, devido às descontinuidades nos campos de tensões e velocidades. Portanto, é natural que os métodos de avanço incremental no tempo mostrem dificuldades na simulação da resposta. Assim, as respostas obtidas nos dois últimos exemplos podem ser consideradas bastante satisfatórias.

Particularmente no que tange à formulação com Dupla Reciprocidade, cabe destacar que nas simulações realizadas bons resultados foram obtidos sem necessidade de introdução de pontos interpolantes no interior do domínio. Disso advém uma vantagem computacional, relacionada às operações com matrizes de menor ordem. Assim, o cálculo de deslocamentos e tensões no interior pode ser feito opcionalmente num pós-processamento.

\section{Agradecimentos}

Aos avaliadores anônimos pela revisão crítica do manuscrito.

\section{Referências}

Bathe K.J. (1996) Finite Element Procedures. New Jersey: Prentice Hall. 1037 p.

Brebbia C.A. \& Dominguez J. (1994) Boundary Elements: An Introductory Course. Southampton: WIT. 322 p.

Brebbia C.A., Telles J.C.F. \& Wrobel L.C. (2012) Boundary Element Techniques. Berlin: Springer. $464 \mathrm{p}$.

Burden R.L. \& Faires J.D. (2008) Análise Numérica. São Paulo: Cengage do Brasil. 736 p.

Cheng A.H.D., Cheng C.S., Goldberg M.A. \& Rashed Y.F. (2001) BEM for thermoelasticity and elasticity with body force - a revisit. Engineering Analysis with Boundary Elements, 25: 377387. https://doi.org/10.1016/S0955-7997(01)00032-7

Guendelman T. (2008) Análise estático y dinámico de estruturas. La Serena: Universidad de la Serena. $236 \mathrm{p}$.

Hetenyi M. (1971) Beams on elastic foundation: theory with application in the fields of civil and mechanical engineering. Michigan: University of Michigan Press. 264 p.

Houbolt J. C. (1950) A recurrence matrix solution for the dynamic response of elastic aircraft. Journal of the Aerospace Sciences, 17: 540-550. https://doi.org/10.2514/8.1722

Katsikadelis J. (2002) Boundary Elements: Theory and Applications. Oxford: Elsevier Science. 448 p.

Klaseboer E., Sun Q. \& Chan D.Y.C. (2019) Helmholtz decomposition and boundary element method applied to dynamic linear elastic problems. Journal of Elasticity, 137: 83-100. https://doi.org/10.1007/s10659-018-09710-y

Lei J., Yun L. \& Zhang C. (2017) An interaction integral and a modified crack closure integral for evaluating piezoelectric crack-tip fracture parameters in BEM. Engineering Analysis with Boundary Elements, 79: 88-97. https://doi.org/10.1016/j.enganabound.2017.04.001

Li J., Shi Z. \& Liu L. (2018) A unified scaled boundary finite element method for transient twodimensional vibro-acoustic analysis of plate-like structures. Computers and Structures, 202 : 105-128. https://doi.org/10.1016/j.compstruc.2018.03.004

Li C., Ooi E.T., Song C. \& Natarajan S. (2015) SBFEM for frature analysis of piezoelectric composites under thermal load. International Journal of Solids and Structures, 52: 114-129. https://doi.org/10.1016/j.ijsolstr.2014.09.020

Malvern L.E. (1969) Introduction to the Mechanics of a Continuous Medium. New Jersey: Prentice-Hall. 771 p.

Meirovitch L. (1975) Elements of Vibration Analysis. USA: McGraw-Hill Int. 495 p.

Morovati V. \& Malek A. (2015) Solving inhomogeneous magnetohydrodynamic flow equations in an infinite region using boundary element method. Engineering Analysis with Boundary Elements, 58: 202-221. https://doi.org/10.1016/j.enganabound.2015.04.021 
Mukhtar F.M. (2017) Relative performance of the three mesh-reduction methods in predicting mode III crack-tip singularity. Latin American Journal of Solids and Structures, 14: 12261250. https://doi.org/10.1590/1679-78253656

Nardini D. \& Brebbia C.A. (1983) A new approach to free vibration analysis using boundary elements. Applied Mathematical Modelling, 7: 157-162. https://doi.org/10.1016/0307-904X(83)90003-3

Nogueira F.C. \& Loeffler C.F. (1991) Resposta dinâmica com o Método dos Elementos de Contorno utilizando Superposição Modal. Revista Militar de Ciência e Engenharia, 8: 36-49.

Partridge P.W., Brebbia C.A. \& Wrobel L.C. (1991) The Dual Reciprocity Boundary Element Method. Berlin: Computational Mechanics Publications. 276 p.

Parvanova S., Vasilev G. \& Dineva P. (2020) Hybrid modelling of multi-layered geological structure under seismic excitation. Journal of Seismology, 24: 183-202. https://doi.org/10.1007/s10950-019-09896-1

Rama B.B. \& Rao V.D. (2001) Advanced Dynamics. Pangbourne: CRC Press. 395 p.

Sayyad A.S. \& Ghugal Y.M. (2016) Single variable refined beam theories for the bending, buckling and free vibration of homogenous beam. Applied and Computational Mechanics, 10: $123-138$.

Trevelyan J. (1994) Boundary Elements for Engineers: Theory and Applications. Southampton: WIT. 228 p.

Vera-Tudela C.A.R. (2003) Formulações Alternativas do MEC para Problemas Elastodinâmicos de Mecânica da Fratura com o uso da Função de Green Numérica. Tese (Programa de PósGraduação em Engenharia Civil). Instituto Alberto Luiz Coimbra de Pós-Graduação e Pesquisa de Engenharia, Universidade Federal do Rio de Janeiro, Rio de Janeiro.

Wang W., Ye W. \& Jiang Y. (2019) A scaled boundary finite element method for bending analysis of fiber-reinforced piezoelectric laminated composite plates. International Journal of Mechanical Sciences, 161-162: 105011. https://doi.org/10.1016/j.ijmecsci.2019.105011

Ye W., Liu J., Lin G., Xu B. \& Yu L. (2018) Application of scaled boundary finite element analysis for sloshing characteristics in an annular cylindrical container with porous structures. Engineering Analysis with Boundary Elements, 97: 94-113. https://doi.org/10.1016/j.enganabound.2018.09.013

Yee K.S. (1966) Numerical solution of initial boundary value problems involving Maxwell's equations in isotropic media. IEEE Transactions on Antennas and Propagation, 14(3): 302307. https://doi.org/10.1109/TAP.1966.1138693

Zhao C., Ailor M. \& Gray L.J. (1994) Interior point evaluation in the boundary element method. Engineering Analysis with Boundary Elements, 13: 201-208. https://oi.org/10.1016/09557997(94)90022-1 